Cahiers d'études italiennes

$21 \mid 2015$

FILIgRANA

L'Italie et l'Orient : échanges, enjeux, regards croisés

\title{
Les cathares de Lombardie et l'Orient : entre sources narratives et documentaires
}

I catari lombardi e l'Oriente. Fonti narrative e documentarie

Luca Fois

\section{OpenEdition}

Journals

Édition électronique

URL : http://journals.openedition.org/cei/2615

DOI : $10.4000 /$ cei.2615

ISSN : 2260-779X

Éditeur

UGA Éditions/Université Grenoble Alpes

Édition imprimée

Date de publication : 5 novembre 2015

Pagination : 23-39

ISBN : 978-2-84310-308-7

ISSN : $1770-9571$

Référence électronique

Luca Fois, "Les cathares de Lombardie et l'Orient : entre sources narratives et documentaires », Cahiers d'études italiennes [En ligne], 21 | 2015, mis en ligne le 01 janvier 2017, consulté le 27 mars 2021. URL : http://journals.openedition.org/cei/2615 ; DOI : https://doi.org/10.4000/cei.2615 


\title{
LES CATHARES DE LOMBARDIE ET L'ORIENT : ENTRE SOURCES NARRATIVES ET DOCUMENTAIRES
}

\author{
Luca Fois \\ Université de Nantes
}

Bien qu'indéniable, le rapport entre les cathares de Lombardie et l'Orient est assez controversér. La lecture des traités qui livrent un récit historique du commencement des communautés dissidentes lombardes — à savoir De heresi catharorum in Lombardia, rédigé entre I2OO et I2I5 par un auteur anonyme, le Tractatus de hereticis, rédigé entre I250 et 1275 par l'inquisiteur Anselme d'Alexandrie, ainsi que, dans une moindre mesure, la Summa de hereticis, rédigée autour de 1250 par l'inquisiteur et ancien hérétique Rainier Sacconi ${ }^{2}$ - ainsi que certains indices disséminés dans d'autres sources, montrent clairement que l'échange avec l'Orient doit être considéré comme un élément clé de leur identité, à partir de ces premières attestations ${ }^{3}$. Il est néanmoins impossible de déterminer à quel point le contenu de ces traités correspond, même vaguement, à des données fondées historiquement. Il est également impossible d'établir à quel point ces données sont le fruit de l'élaboration du phénomène hétérodoxe à travers le prisme de la culture cléricale dans le cadre d'une stratégie précise de

I. Nous utilisons dans ce travail les termes «cathar(es)/catharisme» selon l'usage courant. La question de la légitimité de l'appellation des déviants dualistes (ou dualistes supposés) du XIII ${ }^{\mathrm{e}}$ siècle avec le nom de cathares est néanmoins débattue. Voir à ce propos A. Vauchez, Les Hérétiques au Moyen Âge. Suppôts de Satan ou chrétiens dissidents?, Paris, CNRS Éditions, 20I4, p. 8-9 et M. Zerner (dir.), L'Histoire du catharisme en discussion. Le "concile» de Saint-Félix (II67), Nice, Centre d'études médiévales, 200I, p. I35.

2. Voir à cet égard les éditions, désormais classiques, de A. Dondaine, «La hiérarchie cathare en Italie. I : Le De heresi catharorum» et "II : Le Tractatus de hereticis d'Anselme d'Alexandrie O. P.», dans Id., Les hérésies et l'inquisition, XII -XIII siècles, Aldershot, Variorum, I990, n. III-IV (avec la pagination originale de Archivum Fratrum Praedicatorum 19 [1949] et 20 [1950]), p. 280-312 et 234-324. Pour la Summa de catharis, F. Šanjek, "Raynerius Sacconi O. P. Summa de Catharis», Archivum Fratrum Praedicatorum, 44, 1974, p. 3I-60.

3. Sur les liens avec l'Orient voir le Liber Suprastella ou Supra Stella (S. Burci, Liber Suprastella, C. Bruschi [éd.], Rome, Edizioni di Storia e Letteratura, 2002), le Liber contra Manicheos de Durand de Osca (C. Thouzellier, Une somme anti-cathare. Le "Liber contra Manichaeos" de Durand de Huesca, Louvain, Bibliothèque de l'université - Publications universitaires de Louvain, I96I). 
définition de l'«adversaire» par l'église romaine et par ses membres pendant le XIII ${ }^{\mathrm{e}}$ siècle, ou si - en adoptant le raisonnement inverse - elles sont le résultat de la création d'un «mythe» des origines par les communautés dissidentes elles-mêmes ${ }^{4}$.

La question est complexe et liée à la naissance et à la diffusion du dualisme en Occident, car les traités italiens/lombards sont les seules sources qui en offrent un récit historique articulé et détaillé; ils ont été utilisés au fil des années comme outil permettant de confirmer la véracité d'autres sources ou pour établir des chronologies ou des hiérarchies, non seulement locales'. L'ancien débat sur l'origine orientale du dualisme diffusé en Europe méridionale a été ranimé il y a vingt ans - notamment en France autour de la charte de Niquinta et du concile cathare de Saint-Félix-deCaraman - , ce qui a permis de remettre en question bien des éléments qui étaient tenus pour acquis, principalement sur la base des recherches d'Antoine Dondaine ${ }^{6}$.

L'objectif de cette contribution est d'apporter de nouveaux éléments à ce débat par une relecture, pour l'instant seulement entamée, du Tractatus de hereticis, que la récente découverte de certains documents de l'inquisition milanaise rend désormais possible.

Après la présentation des passages textuels qui contiennent des références à l'Orient, nous proposerons une brève définition de l'Orient dont il est question dans ces textes, menée exclusivement du point de vue géographique. Nous présenterons ensuite quelques remarques sur l'importance du Tractatus de hereticis en comparaison aux sources précédentes ${ }^{7}$ et

4. Ou, encore, s'il s'agit d'un mélange de tout cela : données réelles, érudition/construction polémique des inquisiteurs, mythologie dissidente. Ce sont, grosso modo, les questions que posait, il y a une dizaine d'années, en reprenant des thèmes fortement débattus, D. Zbíral, «La Charte de Niquinta et les récits sur les commencements des églises cathares en Italie et dans le Midi ", Heresis, Carcassonne, Centre national d'études cathares, 44-45, 2006, p. I48: "On peut renouer avec l'historiographie antérieure et conclure que les récits sur les commencements des groupes dissidents dans le De heresi catharorum et le Tractatus de hereticis ne sont pas purement historiques. Ils ont été produits ultérieurement dans un processus de fabrication des mémoires. Le doute subsiste quant à savoir quels fragments de ces narrations peuvent ou ne peuvent pas être reconnus comme historiques; la réponse est — et restera — subjective. Il est néanmoins probable que ces légendes des polémistes aient incorporé des éléments historiques et des fragments de la mythologie dissidente sur les origines telle qu'elle est née au XIII ${ }^{\mathrm{e}}$ siècle quand les circonstances ont poussé les dissidents à se forger des identités plus précises. "Voir aussi les considérations de R. I. Moore, The War on Heresy, Cambridge, Harvard University Press, 2012, p. 322-323.

5. À partir des réflexions de Dondaine, «La hiérarchie cathare», II. Sur le danger de compiler des catalogues d'hérétiques et d'institutions hérétiques, comme sur celui d'une utilisation tautologique des sources du catharisme lombard, voir les remarques de Jacques Chiffoleau dans L'Histoire du catharisme en discussion, ouvr. cité, p. $50-52$.

6. Pour le résumé des positions du débat sur la charte de Niquinta, voir J. Dalarun, «La Charte de Niquinta : débats heuristiques, enjeux herméneutiques", Aevum, vol. 86, nº 2, Milan, Vita e Pensiero, 20I2, p. 535-548.

7. Sans oublier les problèmes encore ouverts sur ce sujet, qui ne peuvent pas par contre être abordés ici. À cet égard nous renvoyons aux remarques formulées par J. Chiffoleau dans L'Histoire du catharisme en discus- 
montrerons enfin, à l'aide de quelques exemples tirés des nouvelles sources documentaires, que le Tractatus de hereticis peut être considéré le fruit de l'activité d'inquisiteur d'Anselme d'Alexandrie et que les informations qu'il contient - y compris, vraisemblablement, les références à l'Orient — ont été construites à la fois sur la base de matériels directs (les interrogatoires des accusés et des récits de ses confrères ex-hérétiques) et indirects (d'autres traités, les registres de ses prédécesseurs, d'autres sources auxquelles il avait accès). Ces informations, bien que presque certainement manipulées et arrangées pour parvenir à un résultat homogène et plausible, ne peuvent pas être considérées in toto comme le produit de la rhétorique antihérétique de l'église et de l'Ordre auquel Anselme appartenait.

\section{Sources narratives : proximité de l'Orient et doutes interprétatifs}

La présence de l'Orient dans les sources narratives sur le catharisme lombard s'enrichit et se structure pendant le XIII ${ }^{\mathrm{e}}$ siècle parallèlement à la croissance de la "conscience historique» de l'existence des hérétiques ${ }^{8}$. Au début, il s'agit d'indications génériques, de caractère presque exemplaire, bien que concrètes et assurément historiques ${ }^{9}$. Par la suite, les références se font progressivement plus réelles : ainsi, les églises dissidentes orientales correspondent à des territoires et à des populations et possèdent des liens et des hiérarchies. Le résultat final est un tableau complet et organique qui permet de dessiner une géographie de la dissidence, d'indiquer ses lignes de développement et ses relations et de définir les étapes de son passage vers l'Occident.

On peut suivre cette évolution dans les textes. Le premier est le De heresi catharorum:

Dans les premiers temps, quand l'hérésie des cathares commença à se développer en Lombardie, ils eurent d'abord comme évêque quelqu'un qui était nommé Marc, et qui gouvernait tous ceux de Lombardie, de Toscane et de la Marche. Ce Marc avait son ordination de Bulgarie. Un certain Papas Nicheta, arrivant en Lombardie, de la région de Constantinople, se mit à contester l'ordination de Bulgarie que détenait

sion, p. 50-55. Plus en général sur l'origine orientale (ou non orientale) du catharisme, voir P. Jimenez-Sanchez, Les catharismes. Modèles dissidents du christianisme médiéval (XII'-XIII siècles), Rennes, PUR, 2008, p. 53-74.

8. G. G. Merlo, Contro gli eretici, Bologne, Il Mulino, I996, p. I25-I52 (et notamment le chapitre intulé : "Coscienza storica della presenza ereticale e della sconfitta degli eretici»). Sur l'apparition de la Bulgarie et des autres régions orientales dans les sources occidentales, voir $\mathrm{M}$. Zerner, «Du court moment où on appela les hérétiques de "bougres». Et quelques déductions", Cahiers de civilisation médiévale, $\mathrm{n}^{\circ}$ I28, Poitiers, CESCM, I989, p. 315-316.

9. G. G. Merlo, Contro gli eretici, ouvr. cité, p. I25-I3I. 
Marc. C'est pourquoi l'évêque Marc commença à hésiter avec ses partisans, puis renonça à l'ordination de Bulgarie et reçut de ce Nicheta l'ordination de Drugonthia. C'est dans cette ordination de Drugonthia qu'il demeura quelque temps avec tous ses complices. Par ailleurs, à un autre moment, vint d'outre-mer, avec ses compagnons, un certain Pétracius, qui apporta des nouvelles à propos d'un dénommé Simon, évêque de Drugonthia, de qui dérivait à l'origine l'ordination que Nicheta avait reçue. Pétracius affirmait que ce Simon avait été surpris avec une femme dans la chambre et qu'il avait fait d'autres choses opposées à la bonne observance. Mais, à l'arrivée de ce Petracius, Marc était déjà mort et un autre, appelé Jean le Juif, qui avait reçu de lui l'ordination épiscopale, lui avait succédé [trad. D. Zbíral]. Et à cause de ce que Pétracius avait dit, certains doutaient de l'ordination reçue par Simon, et d'autres non. Et pour cette raison une dispute surgit parmi eux et ils se divisèrent en deux parties. [...] une partie obéissait à Jean le Juif et l'autre élut comme évêque Pierre de Florence. Et les deux parties restèrent ainsi divisées pendant quelques années [trad. L. Fois] ${ }^{\mathrm{IO}}$.

Dans la suite du récit, les deux parties évoquées ci-dessus essaient de parvenir à un règlement par le biais d'un arbitrage confié à un représentant d'une communauté française; ce dernier conseille de procéder à l'élection d'un nouveau représentant et de l'envoyer en Bulgarie pour y recevoir le consolamentum et l'ordination. En dépit des résistances, le nouveau représentant — nommé Garattus - est élu, et les fidèles fixent un délai pour récolter l'argent nécessaire au financement de son voyage. Néanmoins, avant l'expiration de ce délai, Garattus est surpris en compagnie d'une femme; son élection est par conséquent contestée, et produit de nouvelles divisions. La communauté originale se sépare donc en créant six communautés distinctes qui élisent chacune son propre représentant et l'envoient en Orient pour y recevoir l'ordination. Les Milanais demeurent fidèles à la Bulgarie, où sera envoyé Jean Juif après que Garattus décida de se retirer; la communauté née à Desenzano envoie quant à elle son représentant en Drugonthia, tandis que les communautés de Mantoue et Vicence envoient les leurs en Esclavonie ${ }^{\mathrm{II}}$.

Les informations livrées par la Summa de catharis, bien que plus étendues et moins génériques par rapport à celles fournies par le De heresi catharorum sont apparemment plus concises :

Les églises des cathares sont seize au total [...] l'église d'Esclavonie, l'église des Latins de Constantinople, et dans le même endroit celle des Grecs, l'église de Philadelphie en Roumanie, l'église de Bulgarie, l'église de Drugontie et toutes sont nées des deux dernières.

IO. A. Dondaine, «La hiérarchie cathare», I, ouvr. cité, p. 306, traduction partielle dans D. Zbíral, «La Charte de Niquinta», ouvr. cité, p. I39-I40.

II. A. Dondaine, «La hiérarchie cathare», I, ouvr. cité, p. 307-308. 
[...] ils sont environ 50 de l'église latine de Constantinople. En outre, ils sont, tous ensemble, environ 500 des églises d'Esclavonie, de Philadelphie, des Grecs, de Bulgarie et de Drugontie.

Une fois, Nazaire, leur évêque — qui était très vieux — devant moi et plusieurs autres a dit que la Vierge était un ange et que Christ n'avait pas assumé une nature humaine mais angélique, c'est-à-dire un corps céleste. Et il a dit qu’il avait reçu cette erreur de l'évêque et du fils majeur de l'église de Bulgarie, il y avait déjà presque 60 ans ${ }^{12}$.

Le Tractatus de hereticis reprend quant à lui les informations des deux narrations précédentes et les intègre dans un récit plus articulé et plus riche en détails, y compris la perspective étiologique du préambule :

Il faut noter qu'il y avait en Perse un homme appelé Manès, qui, tout d'abord, s'était demandé : s'il y a un Dieu, d'où vient le mal? S'il n'y a pas un Dieu, d'où vient le bien? Ainsi, il affirma deux principes. Et il enseigna dans les régions de Drugontie, de Bulgarie et de Philadelphie; et dans ces régions l'hérésie s'était tellement répandue que trois évêques furent créés : un de Drugontie, un autre de Bulgarie et un autre de Philadelphie. Ensuite, les Grecs de Constantinople, qui sont à trois jours de voyage des confins de la Bulgarie, y allèrent pour leur commerce et, de retour dans leur pays, comme ils se multiplièrent <dans l'hérésie>, ils créèrent un évêque, qu'on appelle l'évêque des Grecs. Après, les Français allèrent à Constantinople pour soumettre ce pays, et y trouvèrent cette secte, et ils se multiplièrent et ils créèrent un évêque, qu'on appelle l'évêque des Latins. [...] Et parce qu'au début les Français furent séduits à Constantinople par les Bulgares, partout en France ils sont appelés hérétiques bulgares [trad. L. Fois]. [...]. Longtemps après, un notaire de France gagna la Lombardie, précisément la région de Concorezzo, dans le comté de Milan, où il rencontra un certain Marc, qui était de la localité dite Colognia, et le corrompit. Ce Marc parla à deux de ses amis, à savoir Jean le Juif et Joseph. Il est à noter que Marc était fossoyeur, Jean tisserand, et Joseph artisan. L'un d'eux se rendit à Milan, à la porte Orientale dite «de Concorezzo», rencontra un ami, un certain Aldricus de Bando, et le corrompit. Et tous les corrompus prirent conseil auprès dudit notaire, qui les envoya à Roccavione - une localité près de Cuneo - où demeuraient les cathares venus de France pour y habiter. Mais l'évêque des hérétiques n’y était pas; il était à Naples. Ils s'y rendirent pour l'y rejoindre. Ils restèrent là un an. Ensuite, après avoir reçu l'imposition des mains, Marc devint diacre. L'évêque envoya Marc à Concorezzo, son pays d'origine, où Marc prêchait indépendamment. À cause de ses prédications, ils se multiplièrent fortement en Lombardie, ensuite en Marche, et après en Toscane. Ensuite vint un certain Papas Nicheta, qui était l'évêque de ceux de Constantinople, et il constata : "Vous êtes si nombreux qu'il convient que vous ayez un évêque." Ils élirent donc Marc évêque et tous ceux de Lombardie, de Toscane et de la Marche lui étaient soumis. Papas Nicheta le confirma. Peu après, Marc apprit que Papas Nicheta n'était pas bien mort. Il voulut donc aller outremer pour recevoir l'ordination épiscopale de l'évêque de Bulgarie. Mais alors qu'il était en Calabre, il rencontra un diacre

I2. F. Šanjek, "Raynerius Sacconi O. P. Summa de Catharis», ouvr. cité, p. 3I-60. 
cathare nommé Hilaire. Celui-ci lui expliqua qu’il était absolument impossible de traverser la mer. C'est pourquoi il se prépara à rebrousser chemin ${ }^{13}$.

Comme on peut le voir, l'Orient dont il est question ici est tout proche de la Lombardie et de l'Italie ou, du moins, pas trop éloigné : il faut en définir les contours. Les informations du De heresi nous amènent dans la partie sud-orientale de la péninsule Balkanique, en nous faisant arriver jusqu’à la mer Noire. Marc - le premier "évêque» italien — avait reçu, nous ignorons comment, l'ordinatio de Bulgaria. Au début du XIII ${ }^{\mathrm{e}}$ siècle, la Bulgarie venait juste de s'émanciper (en II85) de l'empire de Byzance, et sous le roi (tsar) Kolajan avait été reconnue (en I203-I204) comme État par le pape Innocent III, qui avait aussi accordé le statut de primat à l'évêque de Tirnovo ${ }^{14}$. Pendant le règne de Kolajan, le territoire bulgare s'étendait le long de la dorsale balkanique et était délimité au nord par le Danube, et au sud par l'empire latin de Constantinople (dont l'expansion avait été enrayée par Kolajan à Adrianople en I205); il incluait également la Thrace et une partie de la Macédoine, et était délimité à l'est par la mer Noire et à l'ouest par le royaume des Serbes ${ }^{15}$. La localisation de Drugonthia, où Nicheta avait reçu son ordination, est en revanche plus floue. L'évêché de Dragovitza était traditionnellement indiqué par les sources byzantines comme suffragant du siège de Thessalonique; toutefois, à partir du XIII ${ }^{\mathrm{e}}$ siècle, la région des monts Rhodopes au sud de Philippopolis (Plodiv) était elle aussi désignée par ce nom ${ }^{\mathrm{I}}$. Quoi qu'il en soit, compte tenu des deux possibles localisations, on peut raisonnablement penser que ce nom se réfere au territoire compris entre les Rhodopes au nord et la mer Égée au sud, situé au nord-est de Thessalonique et au sud-ouest de Plodiv. Le troisième élément géographique présent dans le De heresi catharorum est lui aussi lié à Nicheta. En fait, sa provenance de la région de Constantinople étend en direction du sud-ouest l'horizon des cathares lombards qui apparait désormais compris dans un triangle dont les sommets sont situés grosso modo dans les villes actuelles d'Istanbul, Varna et Thessalonique. Après les divisions entre les communautés, l'espace de référence des cathares lom-

I3. A. Dondaine, "La hiérarchie cathare», II, ouvr. cité, p. 308-309, traduction (sauf la première partie) de D. Zbíral, «La Charte de Niquinta», ouvr. cité, p. I40.

I4. M. Zerner, "Du court moment", ouvr. cité p. 3IO-312; L'Histoire du catharisme en discussion, ouvr. cité, p. I22. En général sur l'évolution historique des Balkans pendant cette période : J. V.A. Fine, The Late Medieval Balkans. A Critical Survey from the Late Twelfth Century to the Ottoman Conquest, Ann Arbour, The University of Michigan Press, $2009^{2}$, p. I-53 (Bulgarie p. IO-I7, 25-32, 54-56).

I5. Voir les cartes dans J. V.A. Fine, The Late Medieval Balkans..., ouvr. cité.

I6. I. Dujčev, "Dragovista-Dragovitia", dans Medioevo Bizantino Slavo, II, Rome, Edizioni di Storia e Letteratura, I968, p. I37-I46 (notamment la p. I4I). 
bards s'étend à l'extérieur de ce triangle en direction du nord-ouest, et se rapproche de la péninsule italienne, par l'ajout de l'église d'Esclavonie. Au début du XIII ${ }^{\mathrm{e}}$ siècle, le terme Sclavonia était utilisé dans les textes comme synonyme de «Dalmatie» et indiquait, plus ou moins, le territoire délimité par la mer Adriatique à l'ouest, par la Drave et le Danube à l'est et compris entre l'Istrie au nord et l'actuelle Macédoine au sud ${ }^{17}$. Dans la perception des sources, il s'agit toutefois d'un espace sujet à des modifications, d'où l'on peut parfois retrancher la Bosnie, souvent considérée comme une région distincte, ou la Dalmatie proprement dite ${ }^{18}$. L'auteur du De heresi catharorum ne se soucie guère de donner une définition plus précise des territoires orientaux en complément de l'indication de leurs noms, qui de toute évidence suffisaient aux lecteurs pour identifier des territoires qu'ils connaissaient. Il faut aussi noter que, malgré l'impression persistante que l'Orient était un artifice polémique/narratif, il est néanmoins un espace accessible aux personnages du récit qui n’hésitent pas à s'y rendre pour renouveler leur ordinatio, en un échange vivant et continu entre Orient et Occident ${ }^{19}$.

La Summa de catharis dessine de manière plus succincte, par le biais d'une liste, la géographie du dualisme oriental. L'élément originel introduit par Rainier Sacconi est l'église de Philadelphie en Romania. Dans les sources occidentales contemporaines de la Summa de catharis le terme de Romania, qui s'était répandu après la $\mathrm{IV}^{\mathrm{e}}$ croisade, reprenait la dénomination classique avec laquelle les Byzantins identifiaient leur empire et probablement indique ici l'empire de Nicée, comme suggère la référence à la ville de Philadelphie ${ }^{20}$. Cette ville (l'actuelle Alaşehir en Turquie) était, au $\mathrm{XIII}^{\mathrm{e}}$ siècle, le siège du thème de Thrakeison — dont le territoire était situé dans la partie occidentale de l'Anatolie, en dehors de la ville de Smyrne qui était resté sous le contrôle des grecs après la prise de Constantinople en $\mathrm{I}_{20}{ }^{2 \mathrm{I}}$. Au milieu du XIII ${ }^{\mathrm{e}}$ siècle, l'Orient cathare s'était donc étendu du noyau originel des Balkans et du littoral Adriatique, en traversant le Bosphore, vers l'Asie Mineure.

I7. Sur la définition de Dalmatie/Croatie, voir F. Šanjek, "Albigeois et "chrétiens" bosniaques", Revue d'histoire de l'Église de France, vol. 59, Paris, Letouzey et Ané, 1973, p. 25I; et sur l'interchangeabilité avec Esclavonie, Id., "Les "chrétiens bosniaques" et le mouvement cathare au Moyen Âge", Revue de l'histoire des religions, vol. I82, $\mathrm{n}^{\circ}$ 2, Paris, A. Colin, I972, p. I35-I38.

I8. J. Chiffoleau dans L'Histoire du catharisme, ouvr. cité, p. I24.

19. A. Dondaine, «La hiérarchie cathare», I, ouvr. cité, p. 307-308.

20. J. Chiffoleau dans L'Histoire du catharisme, ouvr. cité, p. I24-I25.

2I. Il faut observer que l'indication de Philadelphie en tant que siège d'une église cathare renvoie immédiatement à l'une des sept églises d'Asie énumérées dans l'Apocalypse de Jean (3, 7-I3). 
Le Tractatus de hereticis reprend les informations des traités précédents et les complète par de nouvelles. En premier lieu, la référence à la Perse — où est située l'origine de tous les catharismes - tout de même excentrique par rapport à l'Orient défini précédemment (il s'agit là de l'élément le plus ouvertement mythique du récit) ${ }^{22}$. En deuxième lieu, le Tractatus contient également des mises au point sur la Bulgarie et l'Esclavonie; il donne ainsi un peu d'épaisseur à la Bulgarie, dont il dit qu'elle se trouve à deux jours de voyage des confins de l'empire d'Orient; il retire en outre la Bosnie (Bossona) de l'ensemble indéterminé de l'Esclavonie, puis la présente comme interchangeable avec elle («episcopum qui dicitur Sclavonie sive Bossone»).

On peut donc entrevoir une évolution dans la perception de l'Orient, qui se fait de plus en plus précise au fur et à mesure que les connaissances des auteurs aussi bien sur les hérétiques que sur l'Orient se font plus importantes. David Zbíral a efficacement montré que les différences entre le De heresi catharorum et le Tractatus de hereticis sont probablement imputables à la tentative d'Anselme d'Alexandrie de construire un récit cohérent et totalisant des événements liés au catharisme lombard par la mythification d'éléments qu'il connaissait bien (à savoir des années I250I270) et par l'effacement de ceux qu'il ne pouvait peut-être pas vérifier ${ }^{23}$. Cela étant dit, on peut noter que ces différences ne sont pas trop marquées pour l'Orient, sinon justement dans le sens d'une plus grande conscience géographique, et par le fait que dans le Tractatus de hereticis l'échange avec les communautés orientales est presque complètement éliminé et limité au seul voyage de Marc, qui, par ailleurs, n'arrivera jamais à destination $^{24}$. En tout cas, l'œuvre d'Anselme d'Alexandrie peut être considérée comme l'aboutissement de la connaissance que l'on avait du phénomène hétérodoxe et de ses liens avec l'Orient, et on peut dire ainsi qu'elle ressortait sans doute de la stratification des informations recueillies par les inquisiteurs dès le début du siècle. En outre, la plus grande qualité et précision des détails présents dans le texte (par exemple sur le voyage de Marc au sud de l'Italie) suggère que le frère avait intégré et vérifié toutes les données par son activité inquisitoriale, en demandant directement aux accusés les informations dont il avait besoin.

22. J. Duvernoy, Le catharisme : la religion des cathares, Toulouse, Privat, 1976, p. 335; J. Chiffoleau dans L'Histoire du catharisme, ouvr. cité, p. 52; D. Zbíral, "La Charte de Niquinta », ouvr. cité, p. I44.

23. D. Zbíral, «La Charte de Niquinta», ouvr. cité, p. I46.

24. Ibid., p. I44. 


\section{L'apport des sources documentaires : un parcours ouvert}

Au-delà de ces considérations, il est difficile d'aller plus loin sans l'appui de sources documentaires permettant d'éclairer l'activité d'Anselme en tant qu'inquisiteur; elles sont nécessaires pour établir un lien entre cette activité et son élaboration dans le traité et, par conséquent, pour juger de la qualité des informations qu'il contient.

Néanmoins de nouvelles découvertes, survenues dans les dernières années et encore en cours d'étude, nous permettent d'orienter quelque peu notre réflexion dans cette direction. Il s'agit en premier lieu d'un groupe de documents notariaux originaux de l'inquisition de Milan provenant des archives dispersées du couvent de Saint-Eustorge, parmi lesquels figurent des documents où Anselme d'Alexandrie agit comme inquisiteur ${ }^{25}$. Presque certainement issues des mêmes archives, se trouvent aussi certaines feuilles de registres inquisitoriaux qu'on peut dater entre les années I250 et I3IO, utilisées à l'époque moderne comme couvertures de certaines liasses de documents notariaux ${ }^{26}$, qui sont en partie également dirigées vers Anselme d'Alexandrie. Il faut rapprocher de ces sources du XIII ${ }^{\mathrm{e}}$ siècle le «cahier» de l'érudit du XviI ${ }^{\mathrm{e}}$ siècle Matteo Valerio sur les hérétiques milanais, conservé à la Bibliothèque nationale Braidense de Milan, découvert et signalé par Paolo Montanari dans les années I990, ensuite utilisé par Marina Benedetti, dont les données peuvent maintenant être, grâce aux nouveaux documents, vérifiées et analysées plus précisément ${ }^{27}$.

Tous ces apports augmentent considérablement les informations dont nous disposons sur le catharisme en Lombardie, non seulement en ce qui

25. Sur la dispersion des archives du couvent de Sant'Eustorgio : L. Fois, «À rebours. Des parchemins milanais de Paris et Halle à la collection oubliée de Giovanni Sitoni (I647-I762)", Bibliothèque de l'École des Chartes, I68, Paris, Droz, 20Io, p. I73-208 (notamment les p. I83-188) et Id., "Documenti milanesi dei secoli XIV-XV nella Bibliothèque nationale de France di Parigi ", Archivio storico lombardo, Milan, Società storica lombarda, 20IO, p. 225-258 (notamment les p. 226-229). Sur les archives de l'inquisition milanaise et leur destruction prétendue, voir L. Fumi, "L'inquisizione romana e lo stato di Milano I», Archivio storico lombardo, Milan, Società storica lombarda, I9IO, p. 8-I6.

26. Ils sont dans une condition forte mauvaise, et la lecture du contenu n'est parfois pas possible. Quoi qu'il en soit, il s'agit de certains dicta testium, d'un registre des condamnations sous forme de rubrique synthétique, et - ce que nous intéresse le plus — d'un livre de sentences appartenant à Anselme d'Alexandrie. Ces documents ne sont pas encore catalogués, ni restaurés; nous tenons donc à remercier ici le personnel de l'ASMi, où le matériel est conservé, pour sa disponibilité.

27. Pour la (re)découverte du cahier de Matteo Valerio, voir les remerciements de Marina Benedetti dans Io non sono Dio. Guglielma da Milano e i Figli dello Spirito Santo, Milan, Edizioni Biblioteca Francescana, I999, p. I2 et Ead., Inquisitori lombardi del Duecento, Rome, Edizioni di Storia e Letteratura, 2008, p. 47. Benedetti (ibid., p. 46-5I) propose des croisements avec les sources qu'elle avait à disposition pour vérifier la fiabilité du contenu du cahier : à savoir le procès des fidèles de Guglielma de Milan, les documents du dossier d'Étienne Confalonieri (les deux dans le ms. BAM, A227 inf.) et les Libri racionum de l'inquisiteur Lanfrancus de Bergame (sur ce dernier voir encore M. Benedetti, Inquisitori lombardi, ouvr. cité, p. 99-I68). 
concerne les données en soi (dates, noms, etc.), mais aussi pour la possibilité d'obtenir une image plus étendue de ce phénomène. Il ne nous est pas possible de proposer ici une relecture complète du Tractatus par le biais de ces nouvelles sources, ni une analyse approfondie de ces dernières; ainsi tenterons-nous de montrer par des exemples à quel point le dialogue entre sources narratives et sources documentaires peut se révéler fructueux pour apporter des corrections, même significatives, à notre connaissance des événements et des croyances des cathares lombards ainsi que, de manière plus générale, pour comprendre les mécanismes de construction des textes des inquisiteurs.

Notre point de départ sera le manuscrit de la Bibliothèque Braidense (dorénavant ms. Braidense ou "cahier»). Il s'agit d'un petit cahier en papier d'environ soixante-dix feuilles, ayant appartenu au prieur de la Chartreuse de Pavie Matteo Valerio. Ce dernier y a noté — parmi d'autres choses des listes de noms de personnes et ajouté quelques brefs passages apparemment - et comme nous le verrons plus loin, presque sûrement - narratifs, tirés de quelques dépositions rendues devant l'inquisiteur ${ }^{28}$. Il s'agit de notes de travail, rédigées par quelqu'un qui avait la possibilité de disposer aisément du matériel originel, dont il copiait les informations qui l'intéressaient, sans devoir se préoccuper de noter systématiquement les données de contexte des documents qu'il utilisait (dates chroniques et topographiques, typologie des documents, etc.) hormis l'indication générique de l'hérésie à laquelle il les attribuait. Malgré cela, l'impression qu'il s'agissait de transcriptions soigneuses, correctes du point de vue paléographique, était déjà ressortie de la comparaison avec les noms présents dans le ms. A227 de la Bibliothèque ambrosienne de Milan — qui contient les documents

28. Le manuscrit contient aux fos $\mathrm{Ir}-2 \mathrm{r}$ des bulles pontificales, aux fos $2 \mathrm{v}-3 \mathrm{v}$ la liste des fidèles de Guglielma, aux $\mathrm{f}^{\text {fos }} 4 \mathrm{rv}$ des listes de cathares, au $\mathrm{f}^{\circ} \mathrm{r}$ une petite liste de fidèles de Dulcin suivie jusqu'au $\mathrm{f}^{\mathrm{o}} \mathrm{I}_{4} \mathrm{v}$ par d'autres listes de cathares (il n'y a plus de spécifications, ni titres, mais il est clair qu'ils sont cathares), au fo isv une liste d'Apostoliques et puis encore de cathares jusqu'au fo $\mathrm{I} 6 \mathrm{r}$, aux fos $\mathrm{I} 6 \mathrm{v}-\mathrm{I} 7 \mathrm{v}$ des membres de familles milanaises, aux

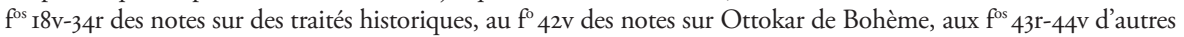
fidèles de Guglielma, aux fos $45 \mathrm{rv}$ d'autres cathares, aux fos $46 \mathrm{r}-67 \mathrm{r}$ d'autres notes historiques sur Milan. La fiche descriptive qui se trouve dans L. Gargan, L'antica biblioteca della Certosa di Pavia, Rome, Edizioni di Storia e Letteratura, 1998, p. I05, récite : «Cart., sec. XVII, mm. 285 x 195, cc. 67 num. rec., bianche le cc. I5a, I8a, 25b, 26a, 27b, 30a, 34b-42a, 48b, 49b-62a, 63, 65a, 66b. Autografo, legatura del sec. XX: piatti ricoperti di carta a fiori, dorso in pergamena con la scritta "MISCELLANE/sic/ DI COSE VARIE"; c. ra inc.: "ex bulla Leonis IX pape...”; c. 67a expl.: “... inter Leonem de Perego archiepiscopum et Martinum Turrianum anno I258, c. 373 " ", en général sur les manuscrits appartenants à Matto Valerio, ibid., p. I03-I08. Le cahier a été recensé aussi par I. Ghiron, Bibliografia Lombarda. Catalogo dei manoscritti intorno alla storia della Lombardia esistenti nella Biblioteca Nazionale di Brera, Milan, Tipografia Bortolotti, I884, p. 137, sous le titre de Valerio (Matteo), Annotazioni storiche diverse. Pour une biographie essentielle de Matteo Valerio (avec la liste de ses ouvrages manuscrits et imprimés), voir P. Argelati, Bibliotheca scriptorum Mediolanensium, II/I, Milan, I745, coll. I553-I555. 
du procès aux fidèles de Guglielma de Milan, la sentence contre Étienne Confalonieri et d'autres témoignages - et par d'autres indices mineurs ${ }^{29}$. Les nouvelles sources à notre disposition confirment cette impression : certains noms des pages des registres correspondent précisément à ceux des listes du "cahier", dont ils décalquent de près même la séquence où ils sont énumérés. C'est par exemple le cas du Lombard Vincemala ainsi que de sa femme Agnès, Vincemala de Vincemalis qui habitaient à Bovisio Masciago au nord de Milan, et d'autres personnes, indiquées dans cet ordre au fo $13 \mathrm{r}$ du "cahier» de Matteo Valerio, tout comme dans celle qui est probablement la page d'un registre des sentences d'Anselme d'Alexandrie $^{30}$. Grâce à ces comparaisons, qui s'ajoutent à celles déjà établies avec le ms. A227 inf., même s'il n'est pas possible de procéder à la vérification de l'ensemble des noms, il nous semble que l'on peut affirmer au moins deux choses : I) le "cahier» de Matteo Valerio est une source fiable, construite sur la base de documents originaux, transcrits par une personne capable de les comprendre parfaitement; 2) si le dossier entre les mains de Matteo Valerio était plus vaste de ce que l'on a imaginé jusqu'ici, il y avait également d'autres documents qu'il ne possédait pas, étant donné que les noms d'hérétiques de certaines des feuilles des registres en notre possession ne sont pas transcrits dans le "cahier ${ }^{3 \mathrm{I}}$.

Revenons maintenant au Tractatus de hereticis. Après avoir vérifié la fiabilité des informations du ms. Braidense et leur précision, on peut immédiatement trouver des pistes intéressantes qui renvoient directement au récit d'Anselme d'Alexandrie. Parmi les nombreux noms des listes, certains reviennent plusieurs fois, suivis de la mention «episcopus catarorum ${ }^{32}$. Sans trop de surprise, ils coïncident pour la plupart avec ceux

29. Gérard de Cambiago : BNB, AE.XII.20, fo 8r. Hubert Maernus/Madernus; fo Iov, Madernus sans spécification; fo IIr, Maernus avec la spécification episcopus hereticorum catarorum (le nom est répété identique pour un autre personnage - Iacobus - au f $\mathrm{f}^{\circ} \mathrm{I} 2 \mathrm{v}$, on peut supposer qu'il s'agit du même document original dont l'information a été prise); $\mathrm{f}^{\circ} \mathrm{I} 4 \mathrm{r}$, Madernus avec la spécification episcopus catarorum.

30. La correspondance est précise (sauf quelques ajustements linguistiques qui ne changent rien au fond), en plus il faut dire que Matteo Valerio n’enregistre que les noms des accusés. Ainsi, dans le ms. Braidense, la liste donne : «Lombardus Vincemala et d. Agnes eius uxor. / Vincemala de Vincemalis de Boisio./ Angelerius de [Quinqueviis] de loco Gradi et Guidottus eius filius» et dans la page du registre d'Anselme l'on retrouve a : I) la condamnation de Lombardus Vincemala de Buisio (datée du 27 août I274), 2) de sa femme Agnès (même date) et 3) celle de Vincemala de Vincemalis (même date) qui avait abjuré, suivies 4) par celle de Anzelerius de Quinque Viis de loco Gradi et probablement par celle de son fils sur le verso du feuillet. Dans l'original se trouvent bien d'autres informations : noms des témoins, des inquisiteurs, des garants, des notaires, la typologie de condamnation (les croix, les amendes, etc.). Bien évidemment, elles n'étaient pas intéressantes pour Matteo Valerio qui ne recherchait que les noms des hérétiques.

3I. Par exemple les feuilles de la rubrique des condamnations où apparaissent au moins I7 noms d'hérétiques condamnés, qui ne sont pas notés dans le cahier.

32. BNB, AE.XII.20, fos 8r, I2r, I4r, I6r. 
de la hiérarchie cathare élaborée par Dondaine sur la base du Tractatus de hereticis ${ }^{33}$. Deux noms en particulier attirent notre attention : ceux de Girardus de Cambiago et de Ubertus Maernus/Madernus. Ils diffèrent sensiblement de la lecture du Tractatus de hereticis, où ils sont rendus, selon l'édition Dondaine, comme Gérard de Cambiate et Hubert Mandennus, mais dans le "cahier» ils sont répétés à l'identique en plusieurs lieux, nous faisant penser qu'il s'agit de leur forme correcte ${ }^{34}$. En fait, on peut ajouter à cet égard qu'il semble beaucoup plus probable qu'une erreur éventuelle soit imputable au copiste allemand anonyme du XIV ${ }^{e}$ siècle, auteur du seul exemplaire connu de l'œuvre d'Anselme d'Alexandrie ${ }^{35}$, plutôt qu'à Matteo Valerio qui pouvait compter sur plusieurs documents originaux du XIII ${ }^{\mathrm{e}}$ siècle et qui avait, par conséquent, la possibilité de vérifier les lectures difficiles et/ou ambiguës.

Ces corrections, bien qu'apparemment minimes, sont importantes parce qu'elles permettent de définir un milieu précis de provenance des personnages les plus importants de l'église de Concorezzo. En effet, s'il est assez difficile, sinon impossible, d'identifier le toponyme Cambiate, la collocation de Cambiago, localité à $\mathrm{I} 2 \mathrm{~km}$ de Concorezzo, est presque immédiate $^{36}$. De même, le nom de famille Maernus/de Maerno, au contraire de Mandennus, est bien attesté dans les sources milanaises de la même époque du Tractatus de hereticis, surtout dans sa variante plus complète de Madernus/de Maderno ${ }^{37}$, qui nous renvoie au lieu de Maderno (actuellement Cesano Maderno), une autre localité située au nord de Milan, assez proche de Concorezzo et encore davantage de Giussano, l'un des lieux plus connus de l'expérience dualiste lombarde ${ }^{38}$. Et de Giussano était originaire Daniel, un autre prélat de l'église de Concorezzo devenu par la

33. Avec Gérard et Hubert on retrouve des autres prélats de l'église de Concorezzo des années I250-I270, BNB, AE.XII.2o, fo $8 \mathrm{r}$ : d. Desiderius flius maior catharorum, Nazarius episcopus catarorum et au fo i2r Henri de Aroxio episcopus catarorum, qui était de l'église de Desenzano.

34. Dans le texte du traité A. Dondaine, "La hiérarchie cathare», II, p. 3Io (Gérard et Hubert) et p. 324 (Hubert), et dans le commentaire de Dondaine, ibid., p. 292-293.

35. A. Dondaine, «La hiérarchie cathare», II, p. 234-235.

36. Dondaine avait avoué la difficulté d'identifier ce Gérard, qui selon lui — avec raison — ne pouvait pas être Girold de Brixia, évêque de la première moitié du siècle («La hiérarchie cathare», II, p. 292-293). Il faut dire qu'une autre hypothèse de lecture du nom de famille, qu'il n'y a aucun support documentaire, pourrait être de Cabiate, un autre petit village auprès de Giussano.

37. Voir par exemple M. F. Baroni (éd.), Gli atti del Comune di Milano nel secolo XIII. Vol. I (I2I7-I250), Milan, Ottavio Capriolo, 1976, ad index (p. 888) la liste des Madernis de, Madernus, qui dicitur Madernus, Maernus.

38. La forme Mandennus présente dans le traité est peut-être une mauvaise transcription : pour quelqu'un qui ne connaît pas les noms (un des copistes médiévaux ou même Dondaine) il est très facile de lire le -r- de Madernus comme un -n-, surtout si le notaire ne sépare pas bien les lettres (-rn- devient -nn-). 
suite inquisiteur ${ }^{39}$, tout comme d'un autre petit village de la même région — Limito - était originaire Pierre, le fils aîné d'Hubert Madernus ${ }^{40}$. Grâce à ces petites corrections, la hiérarchie de l'église de Concorezzo qui était vague (ou plutôt, impossible à déterminer) devient toute de suite beaucoup plus cohérente et plausible par comparaison à une série de noms qui n'avait pas de correspondance dans les sources milanaises. Elle désigne en effet l'existence d'une région précise d'enracinement des hétérodoxes dans le nord/nord-est de Milan, comprise grosso modo entre le fleuve Adda à l'est et le fleuve Seveso à l'ouest, d'où provenaient et/ou agissaient les personnages principaux et leur familles ${ }^{4 \mathrm{I}}$.

En tout cas, la donnée la plus significative qui ressort de ces corrections concerne la méthode de travail d'Anselme d'Alexandrie et les modalités de construction de son traité. La précision des citations (hommes, situation, lieux) dans le texte, aisément vérifiables par quiconque pouvait accéder aux documents de l'officium milanais (et maintenant vérifiables et vérifiées même par nous), nous suggère que le récit historique du Tractatus de hereticis est bien enraciné dans l'expérience inquisitoriale du frère prêcheur, qui, vraisemblablement, recueillait directement des accusés les informations dont il avait besoin. Cela est particulièrement vrai pour les années et les endroits les plus proches de lui (à savoir Milan et son territoire et l'église de Concorezzo) dont il semble avoir une connaissance très étendue et approfondie. Tout cela pouvait d'ailleurs être déjà remarqué par le texte du traité, où plusieurs indices nous donnent des indications sur la provenance des informations mentionnées, en particulier pour les parties où sont exposées les idées des hérétiques, même si de tels indices doivent être interprétés avec prudence ${ }^{42}$. Les accusés n'étaient pas toutefois la seule source d'informations à disposition d'Anselme d'Alexandrie

39. Comme frère Miranus de Cambiago — peut-être parent de Gérard — et Rainier de Placentia "qui tunc erat frater <sic pour filius> minor catarorum et nunc est ex ordine Predicatorum ", les deux cités dans le "cahier" de Matteo Valerio (respectivement BNB, AE.XII.20, fos $8 \mathrm{r}$ et $45 \mathrm{r}$ Miranus et fo $8 \mathrm{r}$ Rainier).

40. A. Dondaine, «La hiérarchie cathare», II, ouvr. cité, p. 293.

4I. De la même provenance - à savoir Buisio/Bovisio Masciago, auprès de Cesano Maderno et Giussano étaient aussi les Vincemala, que nous avons vu cités dans les registres d'Anselme, tout comme Angelerius de Quinque Viis était de Agrate Brianza, un bourg de la paroisse de Vimercate, très proche de Concorezzo. On peut y ajouter aussi Henri de Arosio (fils mineur de l'évêque Bonaventura, de l'église de Desenzano) : Arosio est un village au nord de Giussano; et Aldricus Gilinguellis de Milan (fils mineur de l'évêque Colaianni de l'église de Mantoue-Bagnolo), qu'on doit corriger avec de Ghiringhellis, un nom de famille présent à Milan et dans son territoire (pour les deux noms : A. Dondaine, «La hiérarchie cathare», II, ouvr. cité, p. 306. Sur l'identification de Aldricus, voir l'hypothèse de Jean-Louis Biget dans L'Historie du catharisme en discussion, ouvr. cité, p. I25).

42. A. Dondaine, "La hiérarchie cathare», II, ouvr. cité, p. 318 : "Hoc notavi ego frater A. et scivi ab illis duabus que steterunt longo tempore inter valdenses et fuerunt de secta ultramontanorum; et post converse fuerunt et faciebant penitenciam in reclusorio apud Albam "; et p. 319: "Hac vero que sequuntur habui et didici a quodam Lugio de secta lombardorum, qui captus fuit Ianuae et conversus fuit». 
pour reconstruire les événements du catharisme lombard. Il pouvait sans doute aussi compter sur les mémoires et les connaissances de frère Daniel de Giussano, ancien hérétique de premier plan de l'église de Concorezzo, ou du moins membre d'une famille au cœur de la dissidence lombarde du milieu du XIII ${ }^{\mathrm{e}}$ siècle ${ }^{43}$, auquel — comme il ressort des documents — il était étroitement lié par la militance commune dans le tribunal milanais ${ }^{44}$, et d'autres confrères qui, selon les données à notre disposition, avaient fait partie du champ hétérodoxe avant de se convertir ${ }^{45}$. On ne peut pas non plus oublier qu'Anselme d'Alexandrie pouvait utiliser le patrimoine documentaire (registres, documents notariaux, etc.) conservé dans le couvent de Saint-Eustorge, qui remontait soit à la décennie $125 \mathrm{O}^{46}$, soit aux années I230, comme le suggère une note dans le "cahier» de Matteo Valerio $^{47}$, et d'autres sources manuscrites - comme la Summa de catharis ou le Secret hereticorum/Interrogatio Iohannis cités dans le traité - auxquels il avait libre accès parce que conservées auprès du même couvent milanais, ou provenant des mains de ses confrères ${ }^{48}$.

Il s'agit là de confirmations à des hypothèses qui ont été déjà formulées, en particulier au sujet de l'inclusion probable dans le récit de passages narratifs d'origine dissidente, qu'il faudra élargir et renforcer au fur et à

43. G. G. Merlo, I signori di Giussano, gli eretici e gli inquisitori, Giussano, 2004; M. Benedetti, Inquisitori lombardi, ouvr. cité, p. 75-95.

44. La collaboration entre Anselme et Daniel ressort clairement des feuilles du registre des condamnations où ils agissent ensemble ou comme témoins l'un de l'autre, comme par exemple dans ce document de I265 (l'un des plus complets) : "Pet[raci]us[fil]ius quondam Iacobi de Beatis de Pergamo qui modo habitat in civitate Mediolani, in porta Horientale, iuravit et simili cautione de libris .xxv. terciolorum [ut Gasparus |Prealonus; fideiussor pro eo Mainfredus qui dicitur Zagni filius quondam Pinamontis de [D] exio porte Horientalis. Pro notario Redulfus de Cas[trono] porte Ticinensis. Testes Martinus filius q[ondam] | item Martini Renzoni et Petrus filius quondam item Petri de [........] et Girardus de Bernate. Actum sub porticu fratrum Predicatorum Sancti Eustorgii coram | fratre Daniele de Gluxiano [et fratre] Anselmo de Alexandria inquisitoris. .mcclXv., die veneris, tercio die ante kallendas iunii, indictione octava. [ $\$$ Traditum per] | Guarnerium de Cira notarium.» L'expérience de Daniel de Giussano était appréciée à Milan : désormais âgé il assiste, parmi les autorités laïques et ecclésiastiques de la ville, à la concession à Pagan de Petrasancta de la faculté de faire appel au pape par l'inquisiteur Guillaume de Acqui le ${ }^{\text {er }}$ décembre ${ }_{289}$ (Universitäts- und Landesbibliothek Sachsen-Anhalt, Halle, Morbio I4, $\mathrm{n}^{\circ} 47$ ).

45. Par exemple Rainier Sacconi: M. Benedetti, Inquisitori lombardi, ouvr. cité, p. 39-73.

46. Le plus ancien document connu est le serment des consuls et habitants de Lecco d'observer les mandats de l'église (3I mai I252), cité par E. Motta, «Per la storia dell'eresia in Lombardia nei secoli XIII-XIV», Archivio storico lombardo, Milan, Società storica lombarda, 1906, p. I69-170 et peut-être une sentence de Daniel de Giussano contre Ferrus Villanus datée de la même année I252. La plupart des originaux sont des années I260, comme le serment du podestà et des hommes de Cixnuscolo (Cernusco Lombardone) du 22 mars I262 (Biblioteca Medicea-Laurenziana, Florence, b. 297, n. 7).

47. BNB, AE.XII.2O, $\mathrm{f}^{\circ} 45 \mathrm{r}$ : "Otto Vicecomes fuisse nominatus credens et fautor hereticorum circa an. I23O usque ad I252." Il s'agit quand même d'une donnée qui semble tirée d'une déposition manipulée et commentée par Matteo Valerio, donc il n'est pas possible de la vérifier.

48. Sur la circulation des manuscrits : M. Benedetti, "I libri degli inquisitori», dans G. G. Merlo, Libri, e altro, Milan, Università degli Studi di Milano - Fondazione Arnoldo e Alberto Mondadori, 2006, p. 15-32. 
mesure que seront disponibles de nouveaux documents, ainsi qu'une étude plus approfondie du Tractatus de hereticis ${ }^{49}$.

Nous estimons cependant pouvoir déjà affirmer qu'il semble peu probable qu'Anselme n'ait pas utilisé sa méthode de travail pour obtenir (ou vérifier) les informations sur l'Orient présentes dans son traité comme il l'avait fait pour les autres informations sur les communautés cathares. Donc, tout en admettant une dimension mythique du récit où l'Orient joue un rôle précis dans l'identification de l'adversaire/hérétique, il est vraisemblable qu'elle ne soit pas à attribuer complètement à une construction polémique tirée des connaissances érudites de l'auteur du phénomène dualiste/cathare ${ }^{50}$.

Cette affirmation n'implique pas l'acceptation de la relation avec l'Orient comme effective, mais seulement d'admettre l'existence parmi les dissidents lombards d'une tradition, jalousement préservée pendant soixante-dix années et plus, concernant l'origine de leurs croyances ou, du moins, concernant la source de la légitimité de leurs ministres; et peut-être des éléments de réalité en elle. De même, cela ne signifie pas éliminer complètement les interventions de l'inquisiteur, qui organisa un récit plausible à partir de la grande quantité de données hétérogènes qu'il avait recueillies, en apportant, même involontairement, sa contribution de connaissances et préjugés ${ }^{\text {II }}$. Il nous semble donc que si l'on peut affirmer que soit les inquisiteurs, soit les hérétiques identifiaient sans doute l'Orient comme lieu d'origine des croyances hétérodoxes, il n'est pas vraiment important de savoir s'il l'était réellement; en effet, la référence à l'Orient, même en tant que lieu mythique, devient elle-même une donnée historique et cela devrait suffire.

\section{Bibliographie}

Argelati Philippi, Bibliotheca scriptorum Mediolanensium, II/I, Milan, I745.

Baroni Maria Franca (éd.), Gli atti del Comune di Milano nel secolo XIII. Vol. I (I2I7-I250), Milan, Ottavio Capriolo, 1976.

Benedetti Marina, Io non sono Dio. Guglielma da Milano e i Figli dello Spirito Santo, Milan, Edizioni Biblioteca Francescana, 1999.

49. D. Zbíral, «La Charte de Niquinta», ouvr. cité, p. I48.

50. G. G. Merlo, Contro gli eretici, p. I25-I3I; J. Chiffoleau dans L'Histoire du catharisme, p. 52.

5I. D. Zbíral, «La Charte de Niquinta», ouvr. cité, p. I46-I47. 
Benedetti Marina, "I libri degli inquisitori», dans G. G. Merlo (éd.), Libri, e altro, Milan, Università degli Studi di Milano - Fondazione Arnoldo e Alberto Mondadori, 2006, p. 15-32.

Benedetti Marina, Inquisitori lombardi del Duecento, Rome, Edizioni di Storia e Letteratura, 2008.

Burci Salvo, Liber Suprastella, C. Bruschi (éd.), Rome, Edizioni di Storia e Letteratura, 2002.

Dondaine Antoine, "La hiérarchie cathare en Italie. I : Le De heresi catharorum", dans Id., Les hérésies et l'inquisition, XII ${ }^{e}$-XIII ${ }^{e}$ siècles, Aldershot Variorum, 1990.

Dondaine Antoine, "La hiérarchie cathare en Italie. II : Le Tractatus de hereticis d'Anselme d'Alexandrie O.P.», dans Id., Les hérésies et l'inquisition, XII ${ }^{e}-X I I I^{e}$ siècles, Aldershot Variorum, I990.

DujČEv Ivan, "Dragovista-Dragovitia», dans Medioevo Bizantino Slavo, II, Rome, Edizioni di Storia e Letteratura, I968, p. I37-I46.

Duvernoy Jean, Le catharisme : la religion des cathares, Toulouse, Privat, I976.

Fine John V. A., The Late Medieval Balkans. A Critical Survey from the Late Twelfth Century to the Ottoman Conquest, Ann Arbour, The University of Michigan Press, $2009^{2}$.

Fors Luca, "À rebours. Des parchemins milanais de Paris et Halle à la collection oubliée de Giovanni Sitoni (I647-I762)", Bibliothèque de l'École des Chartes, I68, Paris, Droz, 20I0, p. I73-208.

Fors Luca, «Documenti milanesi dei secoli XIV-XV nella Bibliothèque nationale de France di Parigi", Archivio storico lombardo, Società storica lombarda, 20IO, p. 225-258.

Fumi Luigi, "L'inquisizione romana e lo stato di Milano I», Archivio storico lombardo, I9IO, p. 5-I24.

Gargan Luciano, L'antica biblioteca della Certosa di Pavia, Rome, Edizioni di Storia e Letteratura, 1998.

Ghiron Isaia, Bibliografia Lombarda. Catalogo dei manoscritti intorno alla storia della Lombardia esistenti nella Biblioteca Nazionale di Brera, Milan, Tipografia Bortolotti, I884.

Jimenez-Sanchez Pilar, Les catharismes. Modèles dissidents du christianisme médiéval (XII'-XIII ${ }^{e}$ siècles), Rennes, PUR, 2008.

Merlo Grado G., Contro gli eretici, Bologne, Il Mulino, 1996.

Merlo Grado G., I signori di Giussano, gli eretici e gli inquisitori, Giussano, 2004.

Moore Robert I., The War on Heresy, Cambridge (Mass.), Harvard University Press, 2012. 
ŠAnJEK Franjo, "Les "chrétiens bosniaques" et le mouvement cathare au Moyen Âge", Revue de l'histoire des religions, vol. I82, n² 2, Paris, A. Colin, I972, p. I3I-I8I.

ŠAnjeK Franjo, "Albigeois et "chrétiens" bosniaques", Revue d'histoire de l'Église de France, vol. 59, Paris, Letouzey et Ané, 1973, p. 25I-267.

ŠanjeK Franjo, «Raynerius Sacconi O. P. Summa de Catharis», Archivum Fratrum Praedicatorum, 44, 1974, p. 3I-60.

Thouzellier Christine, Une somme anti-cathare. Le "Liber contra Manichaeos" de Durand de Huesca, Louvain, Bibliothèque de l'université Publications universitaires de Louvain, 196I.

Vauchez André, Les Hérétiques au Moyen Âge. Suppôts de Satan ou chrétiens dissidents?, Paris, CNRS Éditions, 2014.

Zbíral David, "La Charte de Niquinta et les récits sur les commencements des églises cathares en Italie et dans le Midi", Heresis, 44-45, Carcassonne, Centre d'études cathares, 2006, p. I35-I62.

Zerner Monique, "Du court moment où on appela les hérétiques de "bougres". Et quelques déductions", Cahiers de civilisation médiévale, $\mathrm{n}^{\circ}$ I28, Poitiers, CESCM, 1989, p. 305-324.

Zerner Monique (dir.), L'Histoire du catharisme en discussion. Le "concile» de Saint-Félix (II67), Nice, Centre d'études médiévales, 200I. 